\title{
Romance and sex before marriage among young women and men in India
}

International Institute for Population Sciences (IIPS)

Population Council

Follow this and additional works at: https://knowledgecommons.popcouncil.org/departments_sbsr-pgy

Part of the Demography, Population, and Ecology Commons, Family, Life Course, and Society Commons, and the International Public Health Commons How does access to this work benefit you? Let us know!

\section{Recommended Citation}

International Institute for Population Sciences (IIPS) and Population Council. 2010. "Romance and sex before marriage among young women and men in India," Youth in India: Situation and Needs Policy Brief no. 34. Mumbai: IIPS. 


\section{Romance and sex before marriage among young women and men in India}

\begin{abstract}
Pre-marital partnerships among youth, including those that do not involve sexual relations, are widely discouraged in India. Indeed, there is a widespread perception that youth do not find opportunities to mix and form romantic relationships and that pre-marital sex is negligible. These kinds of perceptions are often cited to argue against the provision of sex or family life education and sexual and reproductive health services to unmarried young people.
\end{abstract}

While several small and unrepresentative studies have noted that sizeable proportions of young people, particularly young men, are sexually active before marriage, ${ }^{\text {abcdef }}$ the lack of rigorous and representative evidence on the levels and patterns of intimate partnerships among youth continues to hamper designing of appropriate policies and programmes to address youth sexual health needs. This policy brief documents the extent of pre-marital romantic and sexual relations experienced by young men and women, and the extent to which pre-marital sexual experiences among young people are informed, safe and wanted.

\section{The study}

Data are drawn from the Youth in India: Situation and Needs study, a sub-nationally representative study undertaken for the first time in India of key transitions experienced by young people in six states of India, namely, Andhra Pradesh, Bihar, Jharkhand, Maharashtra, Rajasthan and Tamil Nadu. These states were purposively selected to represent the different geographic and socio-cultural regions within the country, and these six states together represent two-fifths of the country's population. The study included a representative survey of young people in both rural and urban settings. Respondents included unmarried women and men and married women aged 15-24 and, in view of the paucity of married men in these ages, married men aged 15-29.
The survey was conducted in a phased manner in the six states, between January 2006 and April 2008. A total of 50,848 married and unmarried young men and women were interviewed in the survey. These included 8,052 married young men, 11,522 unmarried young men, 13,912 married young women and 17,362 unmarried young women. This brief is based on data obtained from 14,281 young men and 31,274 young women aged 15-24.

Acknowledging that young people may have been reluctant to disclose pre-marital sexual experiences, the Youth Study included a number of approaches to elicit data on sexual behaviour. Questions were posed both in a face-to-face interview format as well as an anonymous format in which youth were asked to mark a blank card indicating whether or not they had ever experienced pre-marital sex, place the card in an envelope, seal it and return it to the interviewer. Envelopes were opened only at study headquarters. In addition, youth were also asked to report, anonymously, the pre-marital sexual experiences of their peers.

Opportunities to form pre-marital romantic relationships do exist for young people Despite norms prohibiting pre-marital opposite-sex mixing, findings indicate that opportunities to form pre-marital romantic relationships did exist for young people. Findings suggest that 23\% of young men and $21 \%$ of young women had either been approached by or had approached a person of the opposite sex to form a romantic liaison. Moreover, 19\% of young men and 9\% of young women acknowledged the experience of a romantic partnership before marriage; rural-urban differences were narrow.

State-wise differences were marked, and regional patterns were not evident. While $22-23 \%$ of young men in Jharkhand, Maharashtra and Tamil Nadu reported the experience of a

\footnotetext{
a Abraham, L. 2001. "Redrawing the lakshman rekha: Gender differences and cultural constructions in youth sexuality in urban India," South Asia 24:133-56.

b Abraham, L. 2002. "Bhai-behen, true love, time pass: Friendships and sexual partnerships among youth in an Indian metropolis," Culture, Health, and Sexuality 4(3):337-53.

c Abraham, L. and K.A. Kumar. 1999. "Sexual experiences and their correlates among college students in Mumbai city, India," International Family Planning Perspectives 25(3):139-46.

d Alexander, M., L. Garda, S. Kanade et al. 2006a. Formation of Partnerships among Young Women and Men in Pune District, Maharashtra. New Delhi: Population Council.

e Alexander, M., L. Garda, S. Kanade et al. 2006b. "Romance and sex: Pre-marital partnership formation among young women and men, Pune district, India," Reproductive Health Matters 14(28):144-55.

f Awasthi, S., M. Nichter and V.K. Pande. 2000. "Developing an interactive STD prevention programme for youth: Lessons from a north Indian slum," Studies in Family Planning 31(2):138-50.
} 
Percentage of youth who reported a pre-marital romantic relationship, according to residence and state

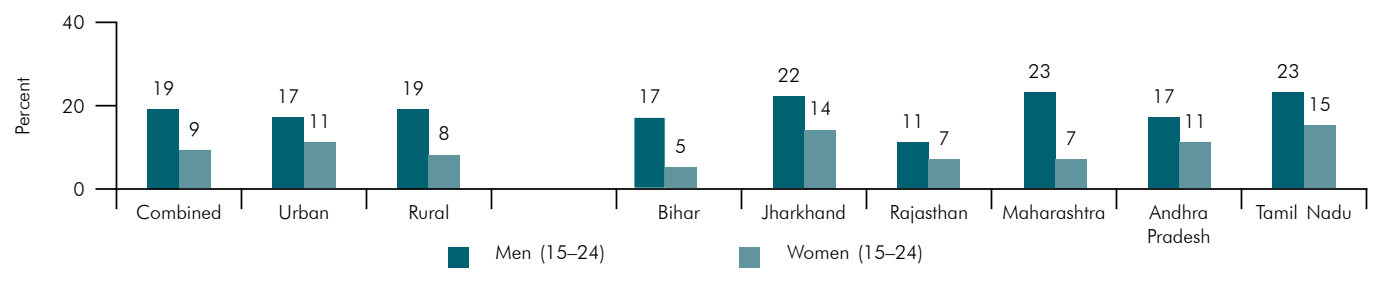

pre-marital romantic partnership, just $11 \%$ of those in Rajasthan so reported. Among young women, those from Jharkhand and the two southern states (Andhra Pradesh and Tamil Nadu) were more likely than those from other states to report a pre-marital romantic partnership (11-15\% and 5-7\%, respectively).

Where romantic relationships occurred, they were usually hidden from parents but not from peers. Among those who had experienced a pre-marital romantic partnership, more than four in five young men and women $(84 \%$ and $82 \%$, respectively) reported that their peers were aware of their relationship; however, only $28 \%$ of young men and $49 \%$ of young women reported that their parents were aware of their relationship.

The majority of young men and women who reported a pre-marital romantic partnership had engaged in a range of intimate behaviours with their romantic partner, from holding hands to kissing on the lips to sexual intercourse. For example, $88 \%$ of young men and $74 \%$ of young women had held hands with their romantic partner. Consistently fewer reported progressively more intimate behaviours: more than two-thirds of young men $(68-71 \%)$ and almost half of young women (46-49\%) had hugged or kissed their romantic partner, and more than two-fifths of young men (42\%) and one-quarter of young women (26\%) had engaged in sex with their romantic partner. It is notable that young women were consistently less likely than young men to report each of these intimate behaviours.

Findings also show that the overwhelming majority of young women $(87 \%)$ engaged in a romantic relationship with expectations of marriage with the romantic partner. Considerably fewer young men who reported a
Progression of physical intimacy among youth who reported pre-marital romantic partnerships

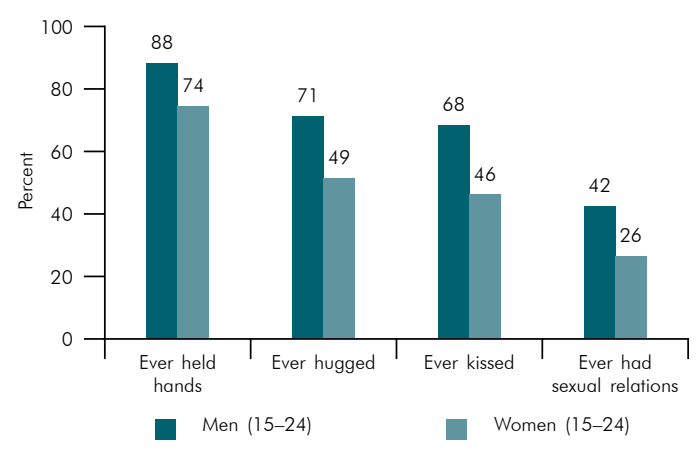

pre-marital romantic relationship expected their romantic relationship to lead to marriage (57\%).

Engaging in sex before marriage is not unknown

The Youth Study inquired about pre-marital sex both with romantic partners and in other situations, for example, casual, paid and forced. Findings indicate that one in seven young men (15\%) and $4 \%$ of young women had engaged in pre-marital sex with romantic and/or other partners. Rural young men were more likely than their urban counterparts to have experienced pre-marital sex (17\% of rural young men compared to $10 \%$ of urban young men). Rural young women were slightly more likely than their urban counterparts to report so (4\% compared to $2 \%$ ). Notably, differences by education were negligible, suggesting that better educated youth were about as likely as their less educated counterparts to have engaged in pre-marital sex.

State-wise differences were not wide, but suggest somewhat different patterns among young men and women. Among young men, similar proportions of those from five of the six states-Andhra Pradesh, Bihar, Jharkhand, Maharashtra and Rajasthan (14-17\%) reported

Percentage of youth who reported pre-marital sexual experiences, according to residence and state

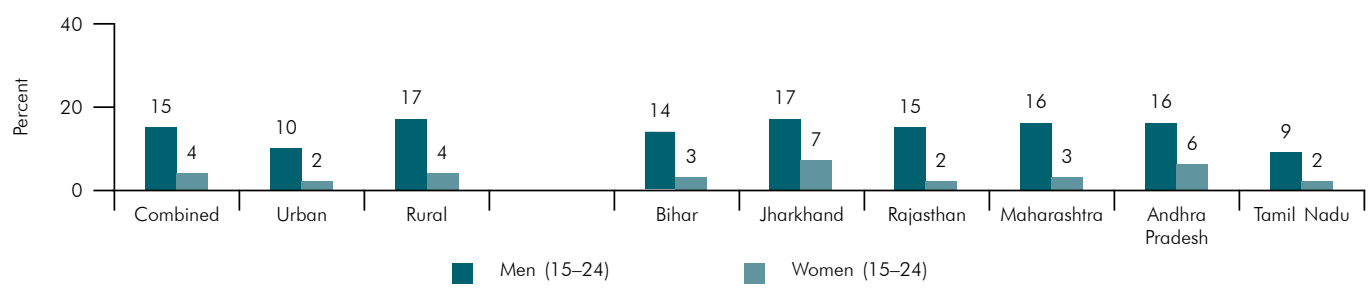


pre-marital sex, compared to considerably fewer of those from Tamil Nadu (9\%). Among young women, those from Jharkhand and Andhra Pradesh were more likely than those from the other states to report pre-marital sex $(6-7 \%$ versus $2-3 \%$ ).

Many young people initiate pre-marital sex uninformed

Findings also confirm that many young people had initiated sexual activity uninformed. For example, just $43 \%$ of young men and $59 \%$ of young women who were sexually experienced before marriage knew that a woman can get pregnant at first sex.

In-depth awareness of contraceptive methods was also somewhat limited, particularly among sexually experienced young women. While 95\% of young men who were sexually experienced before marriage had heard of condoms, fewer (85\%) knew that one condom can be used for only one sexual act. Among young women, the corresponding percentages were 76 and 39 .

Likewise, awareness of HIV/AIDS was limited among sexually experienced youth. While the majority of sexually experienced young men had heard of HIV (90\%), only $43 \%$ of sexually experienced young men reported comprehensive knowledge of HIV/AIDS. ${ }^{1}$ The level of awareness of HIV/AIDS was far lower among sexually experienced young women: only $75 \%$ had ever heard of HIV/AIDS and just 29\% had comprehensive knowledge of HIV/AIDS. Even fewer youth had heard of STIs other than HIV: $26 \%$ of young men and $18 \%$ of young women.

Corresponding with the lack of awareness was lack of exposure to sexuality or family life education. Indeed, just $15-16 \%$ of sexually experienced young men and women had ever received sexuality or family life education.
Engaging in pre-marital sex with multiple partners is not uncommon

Of those who had experienced pre-marital sex, percentage of youth who reported multiple partnerships and consistent condom use

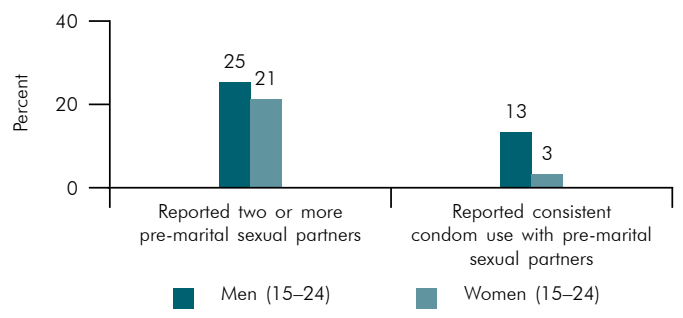

Findings show that where pre-marital sex was experienced, it was by and large unsafe. Of young men who were sexually experienced before marriage, one-quarter had sex with two or more partners. While fewer young women reported that they had engaged in pre-marital sexual relations, one-fifth $(21 \%)$ of these young women reported multiple partners.

\section{Condom use within pre-marital sexual} relationships is almost non-existent Consistent condom use was almost non-existent. Among youth who had experienced pre-marital sex, only $13 \%$ of young men and $3 \%$ of young women reported that they had always used a condom.

Of those who had experienced pre-marital sex, percentage of youth who reported non-consensual sexual experiences

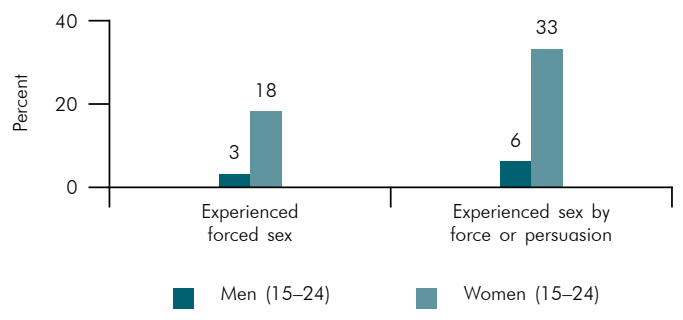

Of those who had experienced pre-marital sex, percentage of youth who reported in-depth awareness of sexual matters

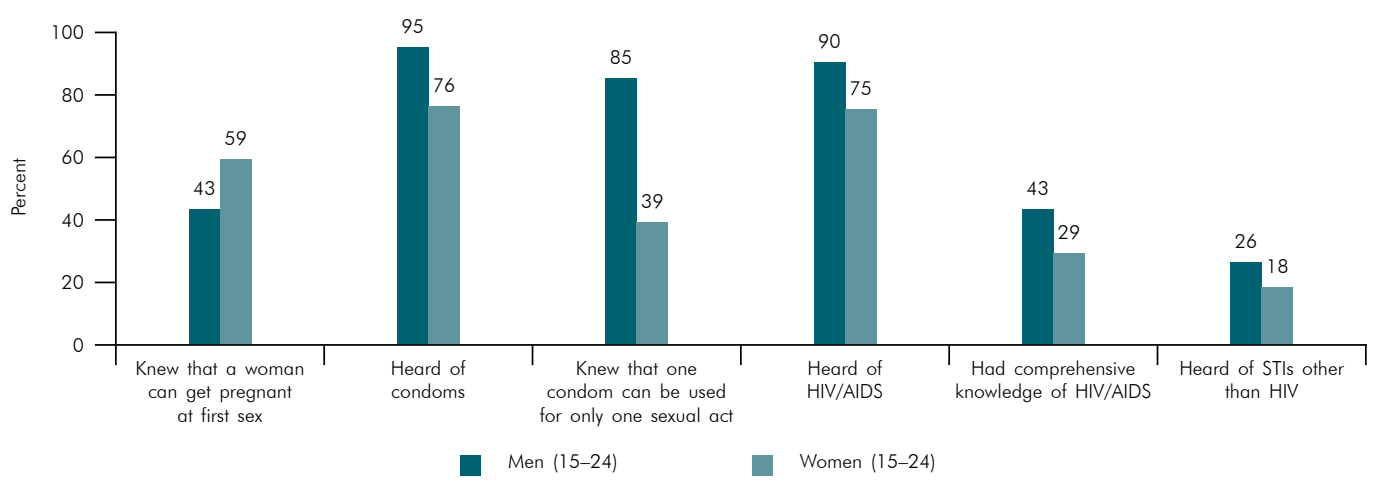

${ }^{1}$ Comprehensive awareness is defined as knowledge of two ways of preventing HIV (specifically, condom use and single partner relations), rejection of common misconceptions about HIV transmission (namely, that HIV can be transmitted through mosquito bites, sharing food or hugging) and awareness that one cannot tell by looking at a person whether he or she has HIV. 
Pre-marital sex is not always consensual Pre-marital sexual experience was non-consensual for substantial minorities of young people, particularly young women. Of those who had engaged in pre-marital sexual relations, $3 \%$ of young men and almost one in five young women $(18 \%)$ reported that they were forced to engage in sex. Few young men $(6 \%)$ and one-third of young women reported that they were either persuaded or forced to engage in sex.

\section{Implications for programmes}

Findings presented above clearly highlight that many young men and a small minority of young women had engaged in sex before marriage and that many of the sexually experienced had initiated sexual activities uninformed, had engaged in unsafe relations and/or had experienced unwanted relations. The better educated were as likely as the less educated to have engaged in sexual relations. Policies and programmes can no longer assume that pre-marital sex does not exist in our culture, rather they must ensure that sexual relations, should they occur, are informed, safe and wanted. Several steps are urgently needed.

Provide family life or sex education for those in school and out of school

The provision of family life or sex education to young people has been a controversial issue in the country. Youth Study findings provide considerable evidence suggesting that family life or sex education is urgently needed among youth, both for those in school and those who have discontinued their education. On the one hand, irrespective of state, few youth had been exposed to family life or sex education. On the other hand, considerable minorities of youth in all states had engaged in unsafe pre-marital sex. These findings emphasise the need for increased investment in promoting such education for youth in school and out of school, and stress that such programmes should be implemented throughout the country and are revived in states in which they have been stalled.

Youth must be equipped with information on physical maturation, relationship matters, risk and protective sexual behaviours (including the role of condoms). They must also be made aware of where they can obtain information, counselling and services. Sex and family life education programmes are needed that build awareness in ways that meet the needs of both those in school and out-of-school, responding to, rather than obfuscating, their questions on sexual health. Such information will allow youth to better assess whether to delay sexual relations, on the one hand and to ensure that relations, if undertaken, are safe, on the other. These programmes should be designed not only to raise awareness among youth but also to enable young people to correctly understand and assess the risks they face and to adopt appropriate protective actions.

Equally important is the acquisition of life skills that enable youth to put information into practice. Findings underscore the need for programmes that focus on building young people's skills in negotiating safe sex and communicating with partners, and that encourage young people to break down gender stereotypes and relate to each other as equals.

\section{Reposition the condom as a suitable method for youth}

Findings have suggested that consistent condom use was rarely practised by those reporting pre-marital sexual relations. At the same time, correct awareness about the condom was far from universal, especially among young women. Moreover, large proportions of youth reported discomfort about seeking contraceptives, including condoms, from a health care provider or pharmacy. Given the appropriateness of the condom for use among young people, it is important that bold and imaginatively designed communication programmes aimed at unmarried as well as married youth are implemented that dispel misconceptions and encourage condom use; and that bold and imaginative changes are made in the service delivery structure that enable youth to access condoms easily and confidentially.

Reorient service provision to address the sexual and reproductive health needs of unmarried youth At the same time, programmes must make available appropriate family planning and infection prevention services for unmarried young men and women in a manner acceptable to them; programmes must recognise their need and right to sexual and reproductive health and related information and services. The National Adolescent Reproductive and Sexual Health strategy of the Reproductive and Child Health $(\mathrm{RCH})$ programme has recognised the need to address the needs of the unmarried, but much remains to be done in terms of reorienting providers to include the unmarried in the ambit of services provided and to shed their own misgivings about addressing the needs of sexually active unmarried youth. At the same time, counselling and contraceptive services must be made available to the unmarried in a non-threatening, non-judgmental and confidential environment. 